\title{
Sentinel-1 SLC preprocessing workflow for polarimetric applications: A generic practice for generating dual-pol covariance matrix elements in SNAP S-1 toolbox
}

\author{
Dipankar Mandal ${ }^{1 *}$, Divya Sekhar Vaka ${ }^{1}$, Narayana Rao Bhogapurapu ${ }^{1}$, \\ V.S. K. Vanama², Vineet Kumar' ${ }^{3}$ Y. S. Rao ${ }^{1}$, Avik Bhattacharya ${ }^{1}$ \\ ${ }^{1}$ Microwave Remote Sensing Lab, Centre of Studies in Resources Engineering, Indian Institute of \\ Technology Bombay, Mumbai, India 400076 \\ ${ }^{2}$ Centre for Urban Science and Engineering, Indian Institute of Technology Bombay, Mumbai, India \\ ${ }^{3}$ Department of Water Resources, Delft University of Technology, Delft, The Netherlands
}

\begin{abstract}
Sentinel-1 SAR data preprocessing is essential for several earth observation applications, including land cover classification, change detection, vegetation monitoring, urban growth, natural hazards, etc. The information can be extracted from the $2 \times 2$ covariance matrix [C2] of Sentinel-1 dual-pol (VV$\mathrm{VH}$ ) acquisitions. To generate the covariance matrix from Sentinel-1 single look complex (SLC) data, several preprocessing steps are required. The ESA SNAP S-1 toolbox can be used to preprocess the data to generate a [C2] matrix. The polarimetric analysis in respective application fields often starts with the covariance matrix. However, due to limited availability of Sentinel-1 SLC data preprocessing workflow standards for polarimetric applications in contemporary research methods, downstream applications unable to comply with these workflows directly. In this paper, we propose a couple of generic practices to preprocess Sentinel-1 SLC data in SNAP S-1 toolbox, which would be beneficial for the radar remote sensing user community.
\end{abstract}

- $\quad$ Single and multi-date data preprocessing workflow for Sentinel-1

- A generic workflow to obtain dual-pol covariance matrix elements from SLC products

- Accurate sub-pixel level coregistration of multi-date data

\section{Keywords}

Sentinel-1, PolSAR, synthetic aperture radar, earth observation, SNAP

\section{Introduction}

Synthetic Aperture Radar (SAR) data in dual polarimetric mode (HH-HV or VV-VH) is widely used in the radar remote sensing community due to its high spatio-temporal coverage. However, compared to a full-pol system, the information content retrieved from a dual-pol system partially characterize a target [1]. Nonetheless, the potential and operational scalability of dual-pol data in various fields such as crop monitoring [2], [3], soil moisture [4], forest biomass [5], land cover classification [6], [7], natural disaster [8] is reported with promising accuracy and reliability. Global data availability and open access data policies of Sentinel-1 A/B dual-pol SAR system lead to the development of operational scale applications [9].

\footnotetext{
* Corresponding Author: Dipankar Mandal (dipankar_mandal@iitb.ac.in)
} 
Much effort has capitalized in the last few years to preprocess Sentinel-1 data for interferometric applications. Majority of the polarimetric studies with Sentinel-1 concentrated on the analysis of backscattered intensities $\left(\sigma_{V V}^{0}\right.$ and $\left.\sigma_{V H}^{0}\right)$ or the ratio of backscattered powers [10], [11]. The phase information between the two orthogonally polarized channels ( $\mathrm{V}$ and $\mathrm{H})$ is inadvertently ignored in these studies. Both the power and phase information is essential to characterize the target in the backscatter field, and forms the rationale behind identifying elementary scatterers in dual-pol mode [12]. The polarimetric information of a target can be represented in form of a $2 \times 2$ covariance matrix ([C2]), which would be helpful for polarimetric analysis. A very few studies indicated the potential of using polarimetric information from [C2] for several applications [13], [14].

To generate a covariance matrix from Sentinel-1 single look complex (SLC) data, several preprocessing steps are involved, which require special attention. European Space Agency (ESA) provides SNAP Sentinel-1 toolbox for processing of these datasets [15]. The toolbox includes several modules for radiometric calibration, coregistration, Sentinel-1 TOPS processing, polarimetry and interferometry, despeckle, geometric correction, etc. [16]. A wide range of end-users requires analysis-ready data (ARD) products (e.g., [C2] matrix elements) for polarimetric analysis with a standard set of corrections and preprocessing workflow. However, there is no standard workflow available in contemporary research methods for preprocessing of Sentinel-1 SLC data for polarimetric applications. In the present method, we propose a couple of generic practices to preprocess Sentinel-1 SLC data in SNAP S-1 toolbox, which would be beneficial for the radar remote sensing user community. The proposed workflows produce a polarimetric matrix from preprocessed Sentinel-1 data, offering a benchmark for the improvement of existing practices.

\section{Imaging geometry and the toolbox}

\subsection{Sentinel-1 imaging geometry}

Sentinel-1 is a constellation of two satellites (1A and 1B) operating in C- band. The constellation reduces the repeat frequency of the satellites from 12 to 6 days; making the data more useful for interferometric and polarimetric applications [9], [17]. Interferometric Wide swath (IW) is one of the acquisition modes of Sentinel-1 capturing data over the land surface. In this mode, three subswaths (IW1, IW2, and IW3) are imaged using Terrain Observation with Progressive Scans SAR (TOPSAR) technique with a spatial resolution of $5 \times 20 \mathrm{~m}$ [18]. The IW products are delivered in form of slices to make the data more manageable in terms of storage and computational effort. Each slice is a stand-alone product and can be processed independently. Each slice covers approximately $250 \mathrm{x}$ $150 \mathrm{~km}$ in range and azimuth direction. The nomenclature of each subswath is based on the orbit type, i.e. ascending or descending. The subswath close to the near range is IW1, and the subswath in the far range is IW3. Each subswath consists of nine bursts in the azimuth direction. The individually focused complex bursts are arranged in azimuth-time order with the black-fill demarcation between them. Figure 1 represents the imaging geometry of a descending pass Sentinel-1 IW product.

\subsection{SNAP Sentinel-1 toolbox}

The Sentinel-1 toolbox (S1TBX) is part of the SNAP software, which supports reading, displaying, processing and writing of data from various remote sensing satellites such as ERS, ENVISAT, Sentinel1, RADARSAT-1 \& 2, PALSAR-1 \& 2, TerraSAR-X, TanDEM-X, COSMO-SkyMed, etc. The toolbox includes several polarimetric and interferometric tools for calibration, coregistration, speckle filtering, subswath merge, subset, multilook and data conversion, etc. The Sentinel-1 toolbox along with other toolboxes (Sentinel-2, Sentinel-3, SMOS, and Proba-V) is widely used by researchers in the field of satellite remote sensing. 

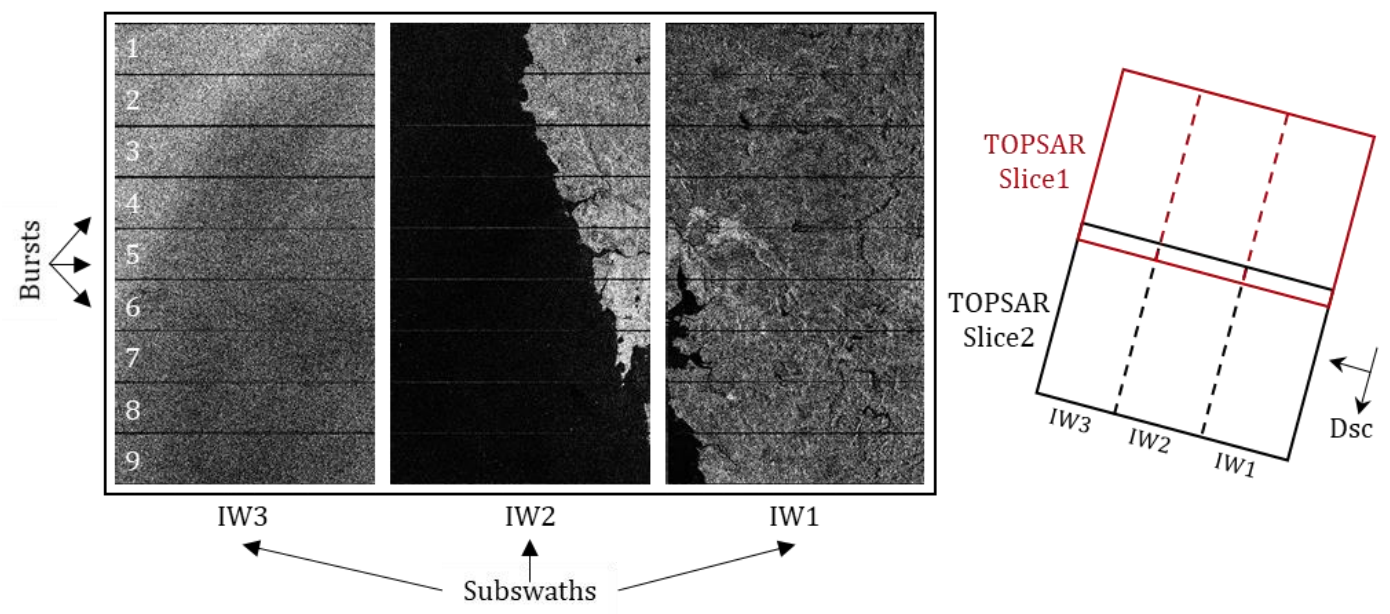

Figure 1: Sentinel-1 descending pass imaging geometry. The red and black rectangles show two individual TOPSAR slices. Each slice has three interferometric wide subswaths (IW1, IW2, and IW3) in range direction and each subswath has nine bursts in the azimuth direction.

\section{Data processing workflows}

In this paper, we describe two essential workflows for Sentinel-1 SLC preprocessing for generating polarimetric matrices. The workflows are 1) Single-date data processing, and 2) Multi-date data processing. These workflows are well tested and computationally efficient. As the name suggests, the first workflow deals with a single data product acquired on a specific date over an area of interest (AOI). However, in the case of temporal analysis or change detection studies, we often perform multidate data processing over an area of interest. Therefore, the second workflow is designed to consider several images, which need to be coregistered to produce accurate and reliable results. In addition to the single or multi-date processing, we describe the processing strategy for three different situations based on the location of AOI.

\subsection{Single-date data processing}

For a single date, the data processing is divided into three categories based on the location of AOI and its coverage by the product swath, as shown in Figure 2. Based on the Sentinel-1 product geometry the categories are: (1) single subswath processing (e.g., IW1 of Slice2) (2) multiple subswath processing (e.g., IW1 and IW2 of Slice2) and (3) assembly of two slices (e.g., IW2 from Slice 1 and 2).

(a)

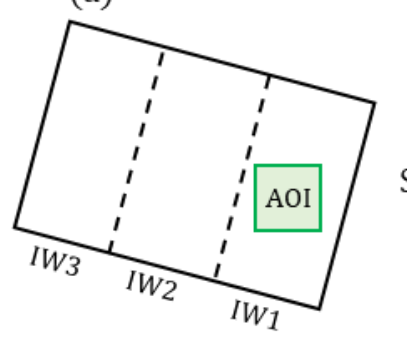

(b)

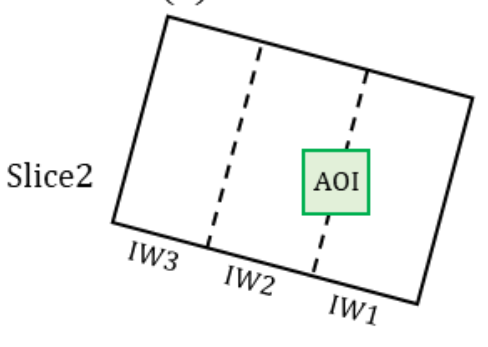

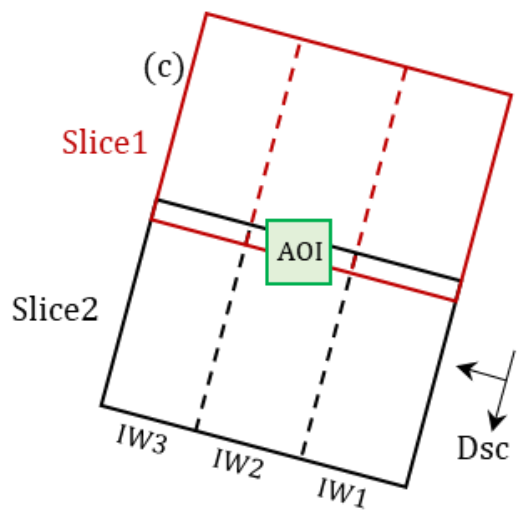

Figure 2: Three main categories of data processing based on the location of users area of interest (AOI). (a) single subswath (b) multiple subswath and (c) assembly of two slices. 


\subsubsection{Category 1: Single swath processing}

For a single subswath (e.g., IW1 of Slice2) processing, the data processing workflow is shown in Figure 3. The workflow consists of four modules. In Module 1, individual Sentinel-1 images are read into the SNAP software, split, a precise/restituted orbit file is applied to update the state vectors, and the image is calibrated. During calibration, it is important to save the output product in complex format. A complex output is necessary to generate a polarimetric matrix in further steps. All the four steps in Module 1 are to be performed individually on each Sentinel-1 image. To save time and to automate the operation, we suggest the user build a graph for Module 1 steps and process. Later, Module 2, 3, and 4 steps are executed one by one to complete single-date data processing. The operators used in these modules are explained in Table 1 . We have provided sample graphs for single and multi-date data processing of a single subswath in the resources link. A user can customize these graphs (.xml files) to suit their processing needs.

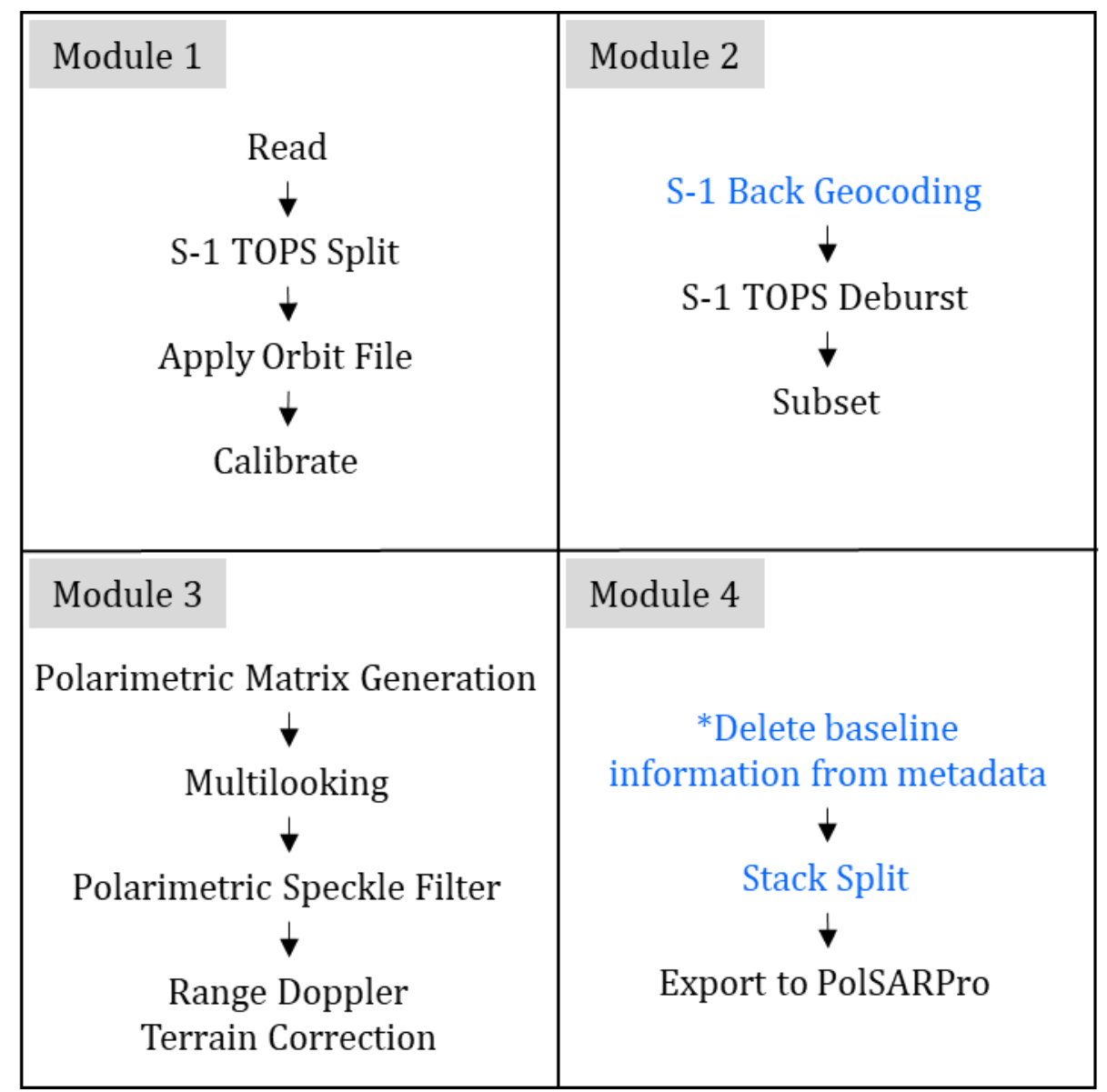

Figure 3: A module-wise flowchart for polarimetric matrix generation. All steps are designed to be performed on a single or a set of images. The steps highlighted in blue are only applicable for multi-date data processing, as described in Section 3.2. The steps indicated by * require manual operation by the user. In the case of multidate data processing, all the steps in Module 1 and the last step in Module 4 is to be performed on individual products.

\subsubsection{Category 2: Multi subswath processing}

Depending upon the category of data processing, we made necessary changes in a specific module, which is to be replaced with an existing module in Figure 3. In case of multiple subswath processing 
(e.g., IW1 and IW2 of Slice2), Module 2 is modified as shown in Figure 4 , which includes the S1-TOPS Merge operator. This operator merges debursted split products of different subswaths into a single image.

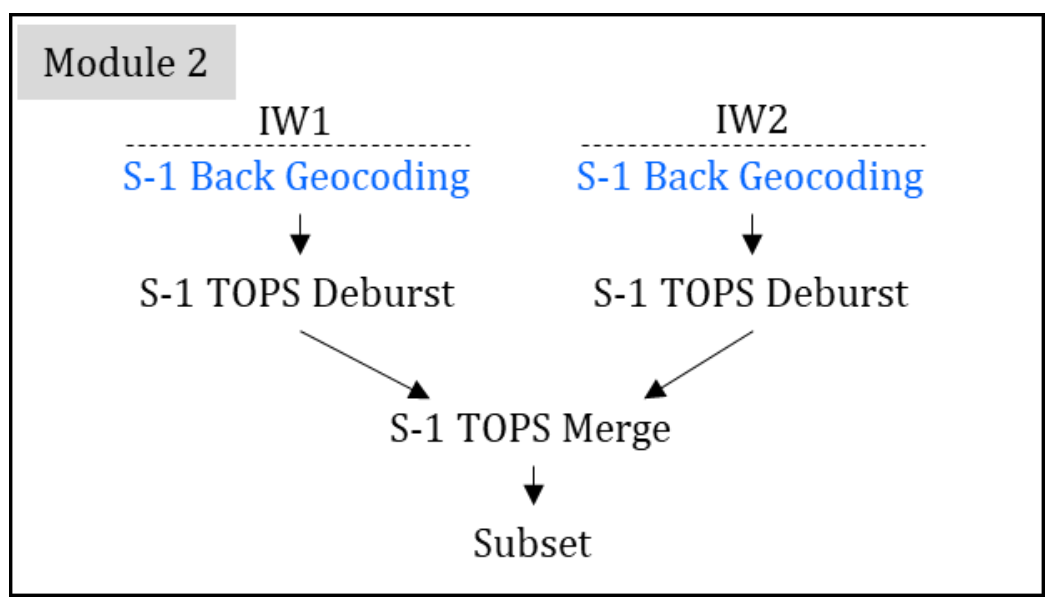

Figure 4: Modification in Module 2 for processing multiple subswaths. Note that S-1 TOPS Merge operator requires input from at least two splits.

\subsubsection{Category 3: Assembly of two slices}

In case of the multiple slice processing of a single date (e.g.: IW2 of Slice 2 and Slice1), Module 1 is modified as shown in Figure 5. The Slice Assembly operator merges two or more slices from different products of an orbit. For example, if AOI lies in between IW2 of Slice 1 and Slice 2 (refer Figure 2c), the Slice Assembly operator assembles the subswaths of different slices of an orbit into a single product.

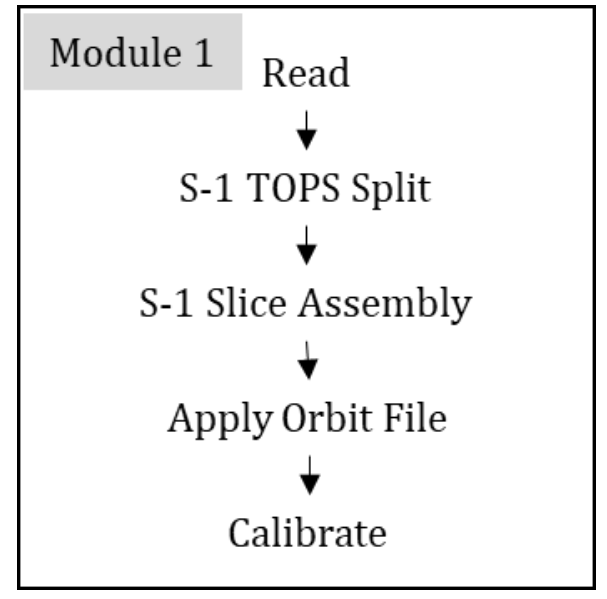

Figure 5: Modification in Module 1 for processing multiple slices.

Module 3 and 4 are the same for the above three processing categories. Module 3 initiates the polarimetric matrix element generation. Here, it is important to note that only $2 x 2$ covariance matrix [C2] is possible from dual-pol Sentinel-1 SLC data. In the presence of quad-pol information (e.g., RADARSAT-2, ALOS-2) it should be changed to $3 \times 3$ covariance [C3] or $3 \times 3$ coherency [T3] matrix. These matrix elements are processed further by despeckling with any of the speckle filters viz., 
Boxcar, Lee, Refined Lee, etc. Selecting the window size is users' choice based on the requirement of feature characteristics [19]. The next step includes an optional choice to geocode the product. At this step, a user should take care of the projection system, e.g., WGS84 or UTM projected coordinate systems.

At the final step in Module 4, the single date data product is exported to PolSARPro format. It stores each matrix elements (C11, C22, C12_real, and C12_imag) individually in a binary format with header information.

\subsection{Multi-date data processing}

It includes processing of SLC data acquired on different dates over an AOI (Figure 6). The multi-date data processing involves coregistration step (S-1 Back Geocoding) from which, a user can generate a stack of coregistered data. The interferometric coregistration step coregisters all the SLC images with sub-pixel level accuracy, which is necessary to produce accurate and reliable results. In the aforementioned single-date processing categories, the steps marked in blue are intended only for multidate data processing. The major difference of this workflow is the co-registration step.
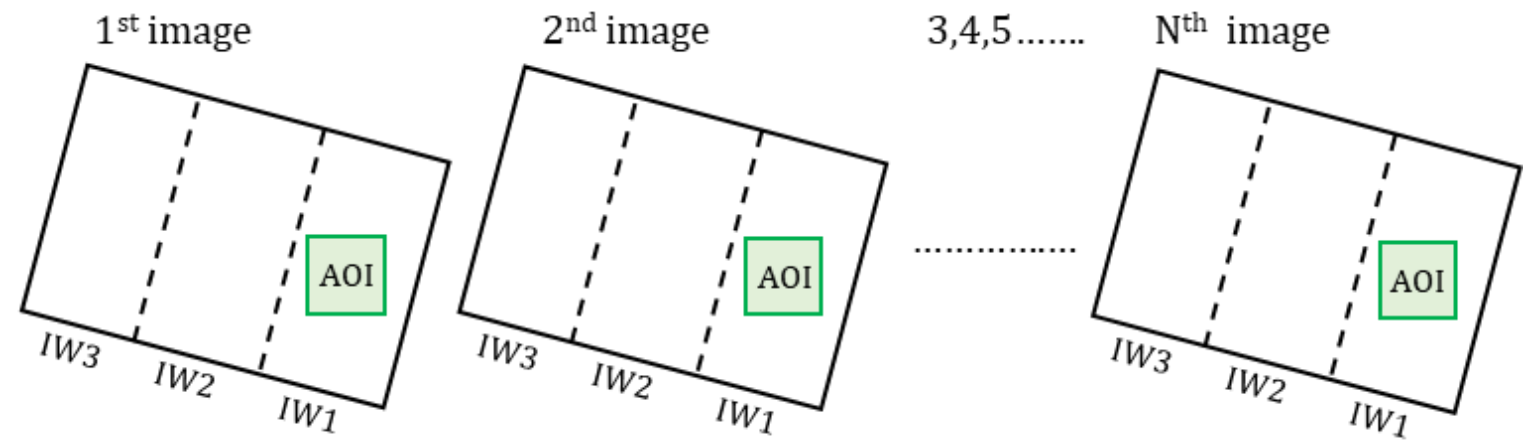

Figure 6: A schematic representation of multi-date images. The AOI represents users area of interest covering in a single subswath.

The S-1 Back Geocoding operator in Module 2 is used to coregister multiple SLC images using a digital elevation model (DEM) and orbit information. A stack consisting of multiple coregistered SLC images is generated after this step. All the bands of an SLC image in the coregistered stack are named based on the image acquisition date. The stack is given as an input to the TOPS Deburst operator, which merges different bursts of an image into a single SLC image. An optional subset operation can be performed on the debursted image to subset the product into a smaller size. A subset operation at this stage is computationally efficient as it reduces the data volume. After this, all the steps in Module 3 are performed on the stacked product.

Another important step for multi-date data processing is shown in Module 4, which deals with baseline information. Due to the implementation of interferometric coregistration, baseline information is present in the metadata of the stacked product. The baseline information has to be removed to export the data from SNAP to PolSARPro format. Therefore, the user has to manually delete the baseline information from the metadata of the stacked product. The stack is then split into individual products using Stack Split operator. Later, these products are exported into PolSARPro format. 
Table 1: Descriptions of different operators and selections in the SNAP platform.

\begin{tabular}{|c|c|c|}
\hline Operator & Description & Operator location in SNAP \\
\hline Read & Read Sentinel-1 IW product & $\begin{array}{l}\text { File }>\text { Import }>\text { SAR Sensors }> \\
\text { SENTINEL-1 }\end{array}$ \\
\hline S-1 TOPS Split & $\begin{array}{l}\text { Split each swath with selected bursts into } \\
\text { a separate product }\end{array}$ & $\begin{array}{l}\text { Radar }>\text { Sentinel-1 TOPS }>\text { S-1 } \\
\text { TOPS Split }\end{array}$ \\
\hline S-1 Slice Assembly & $\begin{array}{l}\text { Merge two or more slices from different } \\
\text { products of an orbit. For example, IW } 2 \\
\text { from Slice } 1 \text { and Slice } 2 \text { as shown in Figure } \\
1 .\end{array}$ & $\begin{array}{l}\text { Radar }>\text { Sentinel-1 TOPS }>\text { S- } 1 \\
\text { Slice Assembly }\end{array}$ \\
\hline Apply Orbit File & $\begin{array}{l}\text { Download and apply precise Sentinel-1 } \\
\text { orbits }\end{array}$ & Radar $>$ Apply Orbit File \\
\hline Calibrate & $\begin{array}{l}\text { Apply the radiometric correction to SAR } \\
\text { images so that the pixel values of the SAR } \\
\text { images truly represent the radar } \\
\text { backscatter of the reflecting surface. } \\
\text { Note: Output to complex data at this step. }\end{array}$ & $\begin{array}{l}\text { Radar }>\text { Radiometric }> \\
\text { Calibrate }\end{array}$ \\
\hline S-1 Back Geocoding & $\begin{array}{l}\text { Coregister two or more SLC products } \\
\text { using orbit files and DEM }\end{array}$ & $\begin{array}{l}\text { Radar }>\text { Coregistration }>\text { S1 } \\
\text { TOPS Coregistration }>\text { S-1 Back } \\
\text { Geocoding }\end{array}$ \\
\hline S-1 TOPS Deburst & $\begin{array}{l}\text { Merge bursts in the azimuth direction to } \\
\text { form a seamless image }\end{array}$ & $\begin{array}{l}\text { Radar }>\text { Sentinel- } 1 \text { TOPS }>\text { S- } 1 \\
\text { TOPS Deburst }\end{array}$ \\
\hline S-1 TOPS Merge & $\begin{array}{l}\text { Merge debursted split products of } \\
\text { different subswaths into a single image. }\end{array}$ & $\begin{array}{l}\text { Radar }>\text { Sentinel- } 1 \text { TOPS }>\text { S- } 1 \\
\text { TOPS Merge }\end{array}$ \\
\hline Subset & $\begin{array}{l}\text { Subset the image as per area of interest. } \\
\text { This step is optional. }\end{array}$ & Raster $>$ Subset \\
\hline $\begin{array}{l}\text { Polarimetric Matrix } \\
\text { Generation }\end{array}$ & Generate polarimetric matrix & $\begin{array}{l}\text { Radar }>\text { Polarimetric }> \\
\text { Polarimetric Matrix Generation }\end{array}$ \\
\hline Multilooking & $\begin{array}{l}\text { Reduce speckle noise and generate a } \\
\text { ground range square pixel }\end{array}$ & $\begin{array}{l}\text { Radar }>\text { SAR Utilities }> \\
\text { Multilooking }\end{array}$ \\
\hline $\begin{array}{l}\text { Polarimetric Speckle } \\
\text { Filter }\end{array}$ & $\begin{array}{l}\text { Despeckle operation on a polarimetric } \\
\text { matrix }\end{array}$ & $\begin{array}{l}\text { Radar }>\text { Polarimetric }> \\
\text { Polarimetric Speckle Filter }\end{array}$ \\
\hline $\begin{array}{l}\text { Range Doppler Terrain } \\
\text { Correction }\end{array}$ & $\begin{array}{l}\text { Geocode the product. This step is } \\
\text { optional. }\end{array}$ & $\begin{array}{l}\text { Radar }>\text { Geometric }>\text { Terrain } \\
\text { Correction }>\text { Range-Doppler } \\
\text { Terrain Correction }\end{array}$ \\
\hline $\begin{array}{l}\text { Delete baseline } \\
\text { information }\end{array}$ & $\begin{array}{l}\text { Delete baselines information manually } \\
\text { from metadata of stacked product }\end{array}$ & \\
\hline
\end{tabular}




\begin{tabular}{|l|l|l|}
\hline Stack Split & $\begin{array}{l}\text { Separate a stacked product into } \\
\text { individual products based on the } \\
\text { acquisition date }\end{array}$ & $\begin{array}{l}\text { Radar }>\text { Coregistration }>\text { Stack } \\
\text { Tools }>\text { Stack Split }\end{array}$ \\
\hline Export & $\begin{array}{l}\text { Export the individual products to } \\
\text { PolSARPro format }\end{array}$ & $\begin{array}{l}\text { File }>\text { Export }>\text { SAR Formats }> \\
\text { PolSARPro }\end{array}$ \\
\hline
\end{tabular}

\section{Summary}

The present study proposes a couple of generic workflows for Sentinel-1 SLC data preprocessing in SNAP S-1 toolbox. The essential workflows to generate a polarimetric matrix are 1) Single-date data processing, and 2) Multi-date data processing. The modular scheme of these workflows allows the user to control the options in each operator. For example, coregistration in rugged terrains can be improved by estimating range and azimuth offsets using enhanced spectral diversity (ESD); or different polarimetric speckle filters can be chosen based on the heterogeneity of the area. The multidate data processing workflow would be of great interest for time series analysis with less error due to the coregistration step. The order of the steps in these workflows is efficiently designed to tackle the memory and data processing issues. Nevertheless, developing a generic workflow for preprocessing steps is important to ensure uniformity across the data sets and results for polarimetric applications.

\section{Acknowledgments}

Authors would like to thank the European Space Agency (ESA) for providing the RSS CloudToolbox service through the ESA EO-SSO users' agreement and the Sentinel Application Platform (SNAP) Sentinel-1 toolbox. The authors are grateful to many individuals working and discussing the development of preprocessing workflows through the ESA STEP forum.

\section{Code availability}

Github .xml codes: https://github.com/mrslab-iitb/sentinel-1-SLC-preprocessing-polarimetry

\section{References}

[1] T. L. Ainsworth, J. Kelly, and J.-S. Lee, "Polarimetric analysis of dual polarimetric SAR imagery," in 7th European Conference on Synthetic Aperture Radar, 2008, pp. 1-4.

[2] J. M. Lopez-Sanchez, J. D. Ballester-Berman, and I. Hajnsek, "First results of rice monitoring practices in Spain by means of time series of TerraSAR-X dual-pol images," IEEE Journal of selected topics in applied earth observations and remote sensing, vol. 4, no. 2, pp. 412-422, 2010.

[3] A. Veloso et al., "Understanding the temporal behavior of crops using Sentinel-1 and Sentinel-2-like data for agricultural applications," Remote Sensing of Environment, vol. 199, pp. 415-426, 2017.

[4] S. Paloscia, S. Pettinato, E. Santi, C. Notarnicola, L. Pasolli, and A. Reppucci, "Soil moisture mapping using Sentinel-1 images: Algorithm and preliminary validation," Remote Sensing of Environment, vol. 134, pp. 234-248, 2013.

[5] J. Reiche, E. Hamunyela, J. Verbesselt, D. Hoekman, and M. Herold, "Improving near-real time deforestation monitoring in tropical dry forests by combining dense Sentinel-1 time series with Landsat and ALOS-2 PALSAR-2," Remote Sensing of Environment, vol. 204, pp. 147-161, 2018. 
[6] D. Bargiel, "A new method for crop classification combining time series of radar images and crop phenology information," Remote sensing of environment, vol. 198, pp. 369-383, 2017.

[7] D. Mandal, V. Kumar, A. Bhattacharya, Y. S. Rao, P. Siqueira, and S. Bera, "Sen4Rice: A processing chain for differentiating early and late transplanted rice using time-series Sentinel-1 SAR data with Google Earth engine," IEEE Geoscience and Remote Sensing Letters, vol. 15, no. 12, pp. 1947-1951, 2018.

[8] S. Plank, "Rapid damage assessment by means of multi-temporal SAR-A comprehensive review and outlook to Sentinel-1," Remote Sensing, vol. 6, no. 6, pp. 4870-4906, 2014.

[9] Z. Malenovskỳ et al., "Sentinels for science: Potential of Sentinel-1,-2, and-3 missions for scientific observations of ocean, cryosphere, and land," Remote Sensing of Environment, vol. 120, pp. 91-101, 2012.

[10] A. I. Flores-Anderson, K. E. Herndon, R. B. Thapa, and E. Cherrington, "SAR Handbook: Comprehensive Methodologies for Forest Monitoring and Biomass Estimation," SERVIR Global, 2019.

[11] V. S. K. Vanama and Y. S. Rao, "Change detection based flood mapping of 2015 flood event of Chennai city using sentinel1 SAR images," in IGARSS 2019-2019 IEEE International Geoscience and Remote Sensing Symposium, Yokohama, 2019, pp. 9729-9232.

[12] D. Ratha, Y. S. Rao, and E. Pottier, "Ambiguities in Poincare sphere orbit signatures of elementary scatterers under orthogonal transformations of the HV basis for dual-pol mode acquisition," presented at the POLINSAR 2019, Workshop on Applications of SAR Polarimetry and Polarimetric Interferometry, Frascati, Italy., 2019.

[13] J. Guo, P.-L. Wei, J. Liu, B. Jin, B.-F. Su, and Z.-S. Zhou, "Crop Classification Based on Differential Characteristics of Halpha Scattering Parameters for Multitemporal Quad-and Dual-Polarization SAR Images," IEEE Transactions on Geoscience and Remote Sensing, vol. 56, no. 10, pp. 6111-6123, 2018.

[14] J. G. Chang, M. Shoshany, and Y. Oh, "Polarimetric Radar Vegetation Index for Biomass Estimation in Desert Fringe Ecosystems," IEEE Transactions on Geoscience and Remote Sensing, vol. 56, no. 12, pp. 7102-7108, 2018.

[15] V. Luis, “Sentinel-1 Toolbox - SAR Basic Tutorial.” European Space Agency, 2016.

[16] “User Guides - Sentinel-1 SAR - Sentinel Online.” [Online]. Available: https://sentinel.esa.int/web/sentinel/userguides/sentinel-1-sar. [Accessed: 18-Sep-2019].

[17] D. S. Vaka, Y. Rao, and A. Bhattacharya, "Surface Displacements of the 12 November 2017 Iran - Iraq Earthquake derived using SAR Interferometry," Geocarto International, 2019.

[18] F. De Zan and A. M. Guarnieri, "TOPSAR: Terrain observation by progressive scans," IEEE Transactions on Geoscience and Remote Sensing, vol. 44, no. 9, pp. 2352-2360, 2006.

[19] L. D. Robertson et al., "SAR Speckle Filtering and Agriculture Field Size: Development of SAR Data Processing Best Practices for the JECAM SAR Inter-Comparison Experiment," in IGARSS 2018-2018 IEEE International Geoscience and Remote Sensing Symposium, 2018, pp. 3828-3831. 